\title{
As abelhas eussociais (Hymenoptera, Apidae) visitantes florais em um ecossistema de dunas continentais no médio Rio São Francisco, Bahia, Brasil
}

\author{
Edinaldo Luz das Neves ${ }^{1}$ \\ Blandina Felipe Viana ${ }^{1}$
}

\begin{abstract}
Highly eusocial bees (Hymenoptera, Apidae) flower visitors in a continental sand dune ecosystem from the medium São Francisco River, Bahia, Brazil. A community of highly eusocial bees in sand dunes, covered with caatinga

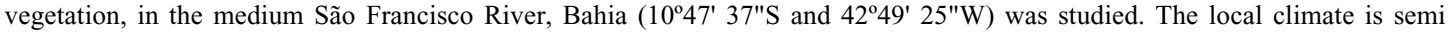
arid and hot, with mean temperature of $25.7^{\circ} \mathrm{C}$ and annual precipitation of $653.8 \mathrm{~mm}$. Censuses took place every two months, from February to December of 2000. The bees were sampled on flowers with entomological nets, from 6:00 a.m. to 5:00 p.m. A total of 2,147 individuals of eight species of Apinae were found, of which Apis mellifera Linnaeus (40.2\%), Trigona spinipes (Fabricius) (28.7\%) and Frieseomelitta silvestri languida Moure (14.7\%) were the predominant species. The diversity was $\mathrm{H}^{\prime}=1.53$ and the evenness $\mathrm{E}^{\prime}=0.73$. The bees were active during the whole year, but there was a significant variation in the monthly abundance of individuals $\left(\chi^{2}=799.55 ; \mathrm{df}=35 ; \mathrm{p}<0.0001\right)$. The daily activity was greater between 6:00 a.m. and 8:00 a.m. The low bee diversity observed is a consequence of the low richness of botanical species and of the small amount of sites for the bees' nests. The community of highly eusocial bees from the dunes presents organization patterns similar to those observed in other caatinga areas, albeit with some particularities.
\end{abstract}

KEYwords: Apidae; Apinae; caatinga; community structure; Meliponini.

\section{INTRODUÇÃO}

As abelhas eussociais (Apidae, Apinae) constituem a principal biomassa de insetos que visitam as flores de vários grupos de plantas em ecossistemas naturais e agrícolas nas regiões tropicais (MichENER 1979; RouBiK 1989; WiLMs et al. 1996). Essas abelhas apresentam longo período de atividade, colônias perenes e sofisticados sistemas de comunicação (SEeley 1985).

Os meliponíneos (Meliponini) têm distribuição pantropical, apresentando grande riqueza de espécies nos trópicos das regiões Neotropical e Indo-Malaia, com alguns táxons restritos às zonas subtropicais do hemisfério sul (MICHENER 1979; ROUBIK 1989). Em levantamentos de abelhas realizados no Brasil, os meliponíneos corresponderam a cerca de $40-75 \%$ dos indivíduos e 5-40\% das espécies coletados nas flores ( RAMALHO 1995; Wilms et al. 1996; CASTRO 2001).

Apis mellifera Linnaeus (Apini) se tornou o visitante floral mais comum nos ambientes tropicais (RouBIK 2000). A diversidade e abundância de Apis mellifera são influenciadas por fatores climáticos, sociais e individuais que afetam os padrões de forrageamento, nidificação e as relações intra-e interespecíficas (CASTRO 2001).

A estrutura da comunidade (composição e fenologia) tem sido o tema preferencialmente abordado no Brasil nos estudos sobre Apoidea (Pinheiro-Machado et al. 2002). Entretanto, poucos compararam seus resultados com os de outros estudos, que utilizaram métodos semelhantes, para testar a existência de padrões entre as comunidades nas diferentes localidades de um mesmo ecossistema e entre ecossistemas distintos, buscando explicações possíveis para os padrões emergentes.

Nos ecossistemas dos mares de areia do médio Rio São Francisco, Viana (1999) estudou aspectos relacionados à diversidade e padrões de abundância das espécies de Apoidea e Neves \& Viana (1999) estudaram a comunidade de machos de Euglossini das matas ciliares próximas aos mares de areia.

$\mathrm{O}$ presente trabalho teve por objetivo investigar se a comunidade de abelhas eussociais das dunas interiores do médio Rio São Francisco (DMRSF) apresenta um padrão de estrutura semelhante ao de outras localidades estudadas na

\footnotetext{
1. Laboratório de Biologia e Ecologia de Abelhas (LABEA), Departamento de Zoologia, Instituto de Biologia, Universidade Federal da Bahia. Rua Barão de Geremoabo s/n, Campus Universitário de Ondina. 40170-110, Salvador - Ba, Brasil. Home page: www.ufba.br/ labea;

Endereço eletrônico: blandefv@ufba.br
} 
caatinga e, mais especificamente:

1. Ampliar o conhecimento sobre a fauna de abelhas eussociais residentes nas dunas, previamente inventariada por VIANA (1999), utilizando uma maior área amostral assim como um maior esforço de coleta;

2. Investigar se há um padrão sazonal (mensal e diário) na atividade de vôo das abelhas, relacionando-o com os fatores ecológicos (recursos alimentares e variações meteorológicas);

3. Verificar a ocorrência de similaridade da fauna de abelhas eussociais das dunas com as de outras localidades estudadas na caatinga;

4. Examinar as principais tendências de organização da comunidade local de abelhas eussociais e propor explicações para as mesmas.

\section{MATERIAL E MÉTODOS}

O estudo de campo foi realizado em uma área restrita de dunas continentais (10 ha), com vegetação de caatinga, próxima ao povoado de Ibiraba ( $10^{\circ} 47^{\prime} 37^{\prime} \mathrm{S}$ e $\left.42^{\circ} 49^{\prime} 25^{\prime} \mathrm{W}\right)$, Bahia, pertencente aos mares de areia do médio Rio São Francisco. $\mathrm{O}$ clima é quente e semiárido (BSwh, segundo o sistema de Köpen), com temperatura média superior a $25,7^{\circ} \mathrm{C}$ (INMET - IV Distrito). As precipitações são escassas e muito irregulares, com média anual de $653,8 \mathrm{~mm}$, apresentando um período seco de sete a oito meses, com as chuvas ocorrendo entre outubro e março (INMET - IV Distrito).

A vegetação local é classificada como caatinga arbustiva aberta, pouco densa em espécies arbóreas e os arbustos e subarbustos são esparsos, apresentando três estratos distintos com predominância do segundo: (1) arbóreo, com representantes de 8-12 m de altura; (2) arbustivo/subarbustivo, com indivíduos de 2-5 m e (3) o herbáceo, geralmente de caráter anual.

As coletas foram realizadas a cada dois meses, durante quatro dias consecutivos, no período de fevereiro a dezembro de 2000. Cada amostragem teve a duração de 11 horas, das 6:00 às $17: 00 \mathrm{~h}$, totalizando $528 \mathrm{~h}$ de esforço amostral, $264 \mathrm{~h}$ por coletor. Vale ressaltar que o esforço de coleta realizado no presente estudo foi maior do que o realizado VIANA (1999) (156h), e as amostragem ocorreram no início (abril), meio (junho) e final (agosto) do período seco e no início (outubro), meio (dezembro) e final (fevereiro) do período de chuvas.

A captura das abelhas nas flores foi feita por dois coletores utilizando redes entomológicas com cabo de $4 \mathrm{~m}$ de altura, segundo método descrito por SAKAGAMI et al. (1967) com algumas modificações. Dois transectos de $500 \mathrm{~m}$ cada, distantes $200 \mathrm{~m}$ um do outro, foram estabelecidos na área. Os mesmos foram percorridos pelos coletores simultaneamente, explorando as plantas floridas a até $50 \mathrm{~m}$ de cada lado do mesmo. O local de coleta foi o mesmo utilizado por Viana (1999), porém o transecto usado por essa autora foi estabelecido no topo de uma das dunas, enquanto os transectos do presente estudo foram estabelecidos cortando três dunas transversalmente, possibilitando a captura das abelhas no topo e nas encostas das dunas e nos vales localizados entre as dunas, além de ampliar a área amostral. O tempo de coleta em cada planta foi de 5 a 10 minutos. Os espécimes coletados estão depositados na Coleção fenológica do Laboratório de Biologia e Ecologia de Abelhas do Departamento de Zoologia do Instituto de Biologia, Universidade Federal da Bahia.

As plantas floridas foram amostradas e prensadas no campo, num total de cinco exsicatas para cada espécie. O número de flores produzido por cada exemplar de espécie vegetal foi estimado por um método arbitrário, contando-se o número de flores contidas em um quadrado de $50 \times 50 \mathrm{~cm}$ colocado aleatoriamente sobre a copa da planta. A estimativa do número de flores por indivíduo florido foi feita utilizando-se a fórmula: $\mathbf{F i}=\mathbf{k . c / q}$ onde $\mathbf{i}$ é o indivíduo florido de determinada espécie vegetal, k é o número de flores contados na área do quadrado, c é a copa total da planta $(\mathrm{m} 2)$ e q a área do quadrado $(0,25 \mathrm{~m} 2)$. Para contagem em plantas com inflorescência, antes de utilizar a fórmula acima, multiplicou-se a quantidade de inflorescências contidas no quadrado pelo número médio de flores por inflorescência. Para a estimativa do número total de flores por espécie botânica no transecto, multiplicou-se o número médio de flores por indivíduo (F) pelo número total de indivíduos floridos na área. Nas plantas de pequeno porte, quando possível, contou-se diretamente o número total de flores. As exsicatas encontram-se depositadas nos herbários RADAM BRASIL (IBGE-Salvador), HUEFS (Universidade Estadual de Feira de Santana, Feira de Santana) e ALCB (Universidade Federal da Bahia, Salvador).

Durante o período amostral, procurou-se aleatoriamente os ninhos de abelhas eussociais presentes na área dos dois transectos (10 ha) e entre os transectos (10 ha). Todos aqueles encontrados foram observados e monitorados.

Nos dias de coleta foram anotados no próprio local, a cada 60 minutos, os dados de temperatura $\left({ }^{\circ} \mathrm{C}\right)$, umidade relativa do ar $(\%)$ e velocidade do vento $(\mathrm{m} / \mathrm{s})$. Os dados de precipitação do dia de coleta, o valor total do mês e os dados da normal climatológica foram obtidos no INMET-IV DisTRITO, referentes ao posto meteorológico do município de Barra $\left(11^{\circ} 05^{\prime} \mathrm{S}\right.$ e $43^{\circ}$ 10 ’W), o mais próximo de Ibiraba.

Para os cálculos de diversidade foi aplicado o índice de Shannon (H') (1949, apud Ludwig \& Reynolds 1988). Para melhor interpretação dos resultados do índice de diversidade, utilizou-se também o índice de eqüitatividade (J') (PIELOU 1975). O programa estatístico utilizado foi o BASIC PROGRAM (LUDWIG \& REYNOLDS 1988).

Para verificar se houve variação significativa na abundância relativa dos indivíduos, durante o período amostral, foi aplicado o teste qui-quadrado $\left(\chi^{2}\right)$. O programa utilizado foi o INSTAT, VERSÃO 3.0 FOR WINDOWS.

As espécies predominantes e seus limites de confiança foram calculados através do método de probabilidade de KATO et al. (1952 apud LAROCA 1995).

Utilizou-se o método de agrupamento UPGMA para avaliar a similaridade entre as faunas de abelhas eussociais das áreas de caatinga estudadas. $\mathrm{O}$ coeficiente de similaridade utilizado 
Tabela I. Número de indivíduos por espécie de abelha eussocial coletados nas DMRSF, no período de fevereiro a dezembro de 2000.

\begin{tabular}{lc}
\hline Espécies de abelhas eussociais (Apidae, Apinae) & $\mathrm{n}$ \\
\hline Apis mellifera Linnaeus, 1758 & 864 \\
Frieseomelitta doederleini (Friese, 1900) & 20 \\
Frieseomelitta silvestrii languida Moure, 1989 & 316 \\
Melipona asilvai Moure, 1971 & 70 \\
Melipona mandacaia Smith, 1863 & 61 \\
Plebeia sp. & 43 \\
Trigona spinipes (Fabricius, 1793) & 615 \\
Trigonisca sp. & 158 \\
\hline Total de indivíduos & 2147 \\
\hline
\end{tabular}

para a aplicação do UPGMA neste estudo foi o MoRISITA-HoRN (MAGURRAn 1988). A matriz de similaridade e o dendrograma foram feitos utilizando-se o programa Multivariate Statistical Package Version $3.12 \mathrm{~d}$.

\section{RESULTADOS}

A riqueza de espécies de abelhas eussociais observadas nas dunas do médio Rio São Francisco (DMRSF) no presente estudo foi semelhante àquela inventariada por VIANA (1999). Estudos taxonômicos realizados por Favízia F. de Oliveira (Departamento de Zoologia, Universidade Federal do Paraná) revelaram que a espécie citada por Viana (1999) como Frieseomelitta sp. refere-se a F. doederleini, e Trigonisca $\mathbf{s p . ~} 1$ e Trigonisca sp. 2 são a mesma morfo-espécie. Esta confere com Trigonisca sp. obtida no presente estudo.

Foram capturados 2.147 indivíduos, representantes de oito espécies de abelhas eussociais da família Apidae, sendo sete espécies (cinco gêneros) da tribo Meliponini e uma espécie de Apini (Tabela I). As espécies predominantes foram A. mellifera, Trigona spinipes (Fabricius 1793) e Frieseomelitta silvestrii languida Moure 1971. A diversidade obtida foi de $\mathrm{H}^{\prime}=1,53 \mathrm{e}$ a eqüitabilidade $\mathrm{J}^{\prime}=0,73$.

A análise de qui-quadrado mostrou que houve uma variação significativa na abundância mensal do número de indivíduos de Apinae $\left(\chi^{2}=799,55 ; \mathrm{gl}=35 ; \mathrm{p}<0,0001\right)$.

A maior quantidade de flores disponíveis no campo foi estimada no mês de fevereiro (estação de chuvas). Contudo, o maior número de abelhas foi capturado em outubro, mês com menor quantidade de flores disponíveis na área, baixo índice de precipitação (Fig. 1), mais alta temperatura média $\left(28,7^{\circ} \mathrm{C}\right)$, menor umidade relativa (43\%) e maior velocidade média do vento $(3,5 \mathrm{~m} / \mathrm{s})$.

A. mellifera foi mais freqüente nas flores no final do período seco. T. spinipes manteve sua atividade uniforme durante todo o período seco, tendo sua atividade bastante reduzida no período de chuvas. Frieseomelitta s. languida foi mais abundante no início do período de chuvas, tendo sua atividade reduzida no auge do período seco.

Durante o período amostral, as abelhas eussociais estiveram mais ativas no intervalo das 6:00 às 8:00h (Fig. 2), quando a temperatura estava mais baixa e a umidade relativa, mais alta (Tabela II).

A. mellifera e $T$. spinipes estiveram ativas durante todo o dia, entretanto mostraram-se mais freqüente nas flores no intervalo das $6: 00$ às $8: 00 \mathrm{~h}$. Frieseomelitta s. languida evidenciou a maior atividade no intervalo das 10:00 às 12:00h e das 14:00 às 16:00h.

Foram contabilizados dois ninhos de A. mellifera, sendo que um dos enxames, implantado em oco de uma Copaifera luetzelburgii Harms (Caesalpiniaceae), abandonou o local no mês de abril e o segundo (aéreo), no galho de um Pseudobombax sp., foi predado por meleiros no mês de dezembro, poucos dias antes da última coleta. Este último apresentou a maior população no mês de outubro, mês que sofreu enxameagem.

Foram observados dois ninhos aéreos de $T$. spinipes, um em C. luetzelburgii e outro em Pseudobombax sp. As demais espécies nidificaram em ocos de $C$. luetzelburgii, sendo três ninhos de Melipona asilvai Moure, 1971 e Frieseomelitta doederleini (Friese, 1900), e um de Melipona mandacaia Smith,

Tabela II. Abundância relativa de abelhas eussociais coletadas e médias de temperaturas e umidades relativas nas DMRSF, nos diferentes intervalos de horas, no período de fevereiro a dezembro de 2000; $\mathrm{dp}=$ desvio padrão.

\begin{tabular}{lccccc}
\hline \multirow{2}{*}{ Horário } & \multirow{2}{*}{ \% indivíduo } & \multicolumn{2}{c}{ Temperatura $\left({ }^{\circ} \mathrm{C}\right)$} & \multicolumn{2}{c}{ Umidade Relativa (\%) } \\
\cline { 3 - 6 } & & Média & $\mathrm{cp}$ & Média & $\mathrm{dp}$ \\
\hline 6:00- $8: 00$ & 36,4 & 25,0 & 1,9 & 71,1 & 6,3 \\
8:00-10:00 & 16,8 & 31,1 & 2,2 & 50,4 & 6,8 \\
10:00- $12: 00$ & 16,3 & 34,7 & 2,1 & 41,4 & 7,5 \\
12:00-14:00 & 10,1 & 36,3 & 2,1 & 38,1 & 7,5 \\
14:00-16-00 & 15,9 & 34,7 & 2,6 & 38,9 & 8,6 \\
16:00-18:00 & 4,50 & 34,3 & 2,9 & 39,0 & 6,9 \\
\hline
\end{tabular}



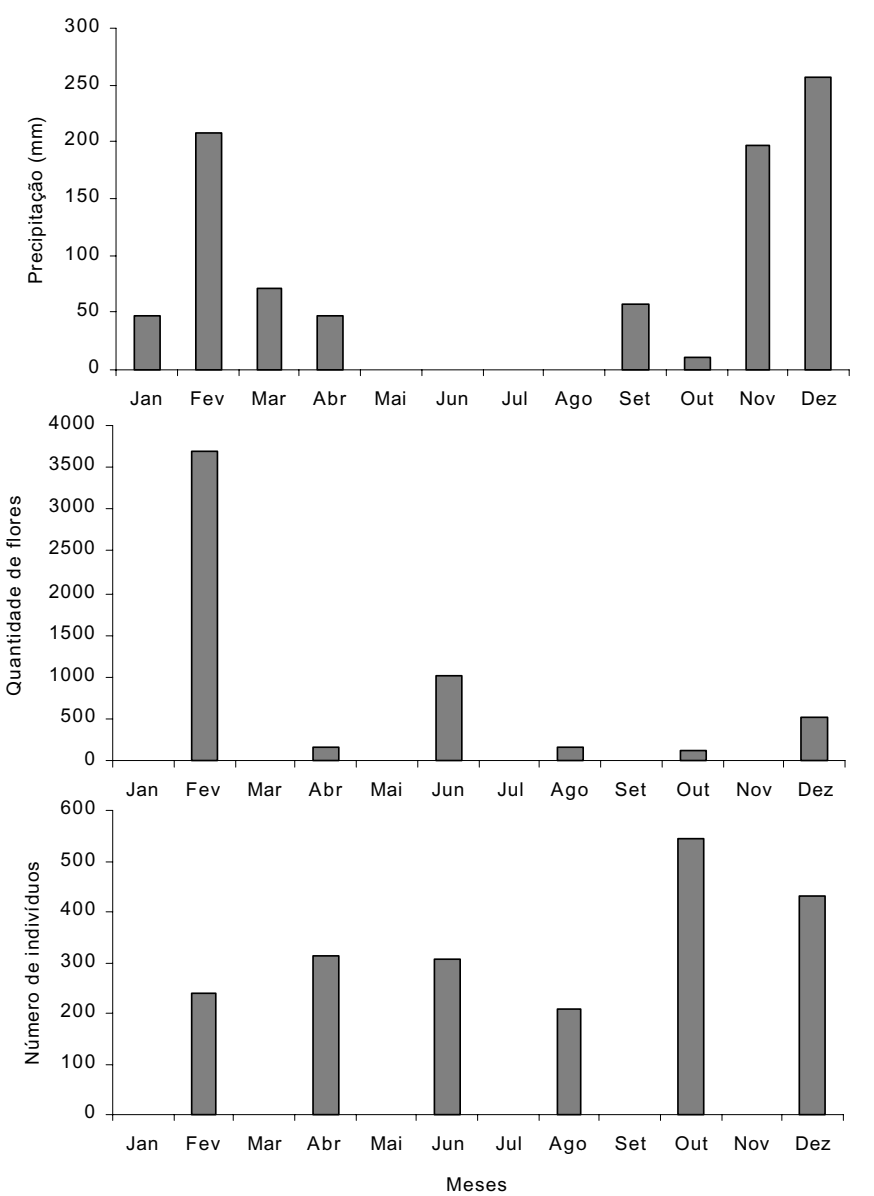

Fig. 1. Variação mensal nos fatores ecológicos (precipitação e quantidade de flores) e no número de indivíduos de abelhas eussociais coletadas a cada dois nas DMRSF, no período de fevereiro a dezembro de 2000.

1863, Plebeia sp. e Trigonisca sp. Todos os ninhos monitorados permaneceram ativos, com entrada e saída de abelhas transportando pólen, durante todo o ano.

\section{DISCUSSÃO}

A riqueza de espécies de abelhas eussociais observadas nas dunas do médio Rio São Francisco (DMRSF), assim como as de outras localidades da caatinga, foi baixa quando comparada a outros ecossistemas brasileiros.

Dentre os levantamentos de abelhas em ambiente de caatinga, nas flores e nos ninhos (CASTRO 1994; Aguiar \& Martins 1997; Carvalho 1999; Viana 1999; Teixeira \& Viana 2002), a maior riqueza de espécies de abelhas eussociais $(\mathrm{n}=19)$ e diversidade $\left(H^{\prime}=1,63 ; \mathrm{J}^{\prime}=0,55\right)$ foi obtida em Milagres-BA, em uma área de ecótono com a mata atlântica (CASTRO 2001), provavelmente devido à facilidade de colonização da área por populações emigrantes do ecossistema vizinho. Os dados obtidos neste estudo, corroboram a hipótese de CASTRO (2001) de que a riqueza de espécies de abelhas eussociais aumenta a partir da área central da caatinga (core) em direção à Mata Atlântica (Fig. 3).
As assembléias de abelhas eussociais da caatinga são caracterizadas pela predominância de $A$. mellifera e $T$. spinipes e por espécies de outros gêneros (p. ex., Frieseomelitta, Melipona, Trigonisca, Plebeia) que se substituem nas diferentes localidades (Fig. 4). As diferenças estruturais locais, as variações meteorológicas e a influência dos ecossistemas vizinhos favorecem a fragmentação da caatinga em hábitats singulares, impedindo a emergência de um padrão de organização generalizado das abelhas eussociais para esse bioma.

Alguns fatores devem estar atuando na organização da comunidade de abelhas eussociais nas DMRSF, favorecendo o atual padrão de baixa diversidade e riqueza de espécies, dentre eles: (1) a recente formação dos mares de areia, disponibilizando pouco tempo evolutivo para a colonização da área pelas espécies de abelhas a partir das fontes colonizadoras (p. ex., Mata Atlântica); (2) a baixa riqueza de espécies vegetais presentes nas dunas. Essa baixa riqueza de espécies botânicas também deve ser resultado do curto período de tempo decorrido a partir do início da colonização das dunas. De acordo com dados geomorfológicos e palinológicos, o processo de fixação das dunas dos mares de areia deve ter iniciado no Pleistoceno, no final da glaciação Würm-Wisconsin (De Oliveira et al. 1999). Esse curto período evolutivo (cerca de 10.540 anos AP) ainda não foi suficiente para que houvesse uma colonização eficiente da área com linhagens adaptadas às condições adversas das dunas, propiciando uma alta riqueza de espécies; (3) a pouca disponibilidade de locais adequados para nidificação, já que algumas espécies desse grupo necessitam de cavidades préexistentes com perímetros superiores a $80 \mathrm{~cm}$, cupinzeiros ou solos adequados para a construção de seus ninhos e/ou (4) as restrições fisiológicas de algumas espécies às condições meteorológicas adversas das dunas.

Apesar do impedimento taxonômico, já que a maioria dos indivíduos do gênero Plebeia, Trigonisca, Frieseomelitta e Partamona não foi identificada até espécie, pode-se sugerir que existe maior similaridade entre a fauna de abelhas das dunas com as faunas do agrupamento Casa Nova e Castro Alves

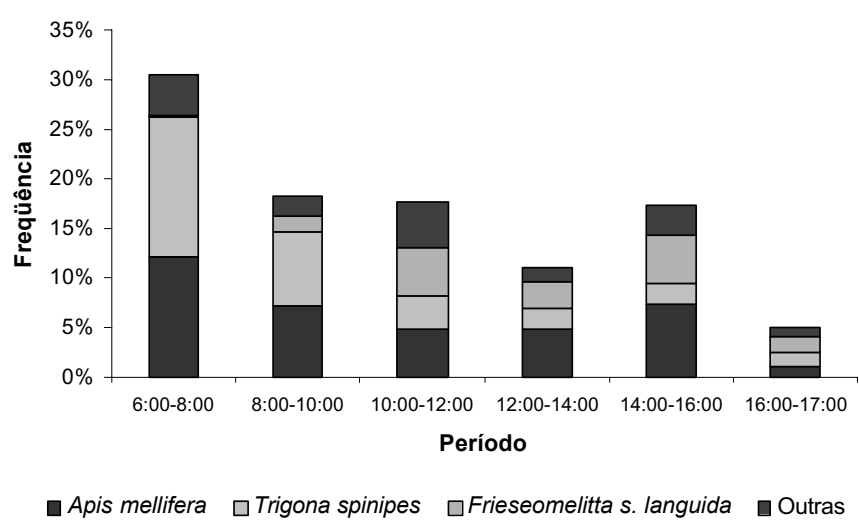

Fig. 2. Atividade diária dos indivíduos de abelhas eussociais predominantes nas DMRSF, no período de fevereiro a dezembro de 2000. 
(Fig. 5). As DMRSF e Casa Nova são as localidades estudadas mais próximas da área nuclear da caatinga e o fato de não apresentarem as faunas mais similares deve ser conseqüência da história evolutiva diferenciada das dunas que lhe conferiu características estruturais exclusivas, tais como a presença de algumas espécies botânicas chaves que disponibilizam recursos florais para as abelhas durante os períodos de escassez de chuva.

Estudos sobre os Apoidea da caatinga revelaram que Apinae é o grupo mais abundante nesse bioma. Nas DMRSF, a maior abundância relativa de $A$. mellifera e $T$. spinipes pode ser atribuída ao fato dessas abelhas (1) possuírem colônias muito populosas (5.000 a 80.000 indivíduos), (2) forragearem até grandes distâncias de seus ninhos, (3) explorarem numerosas espécies de plantas, (4) possuírem sistemas de comunicação muito eficientes e (5) não dependerem de cavidades préexistentes para nidificação.

Apis mellifera tem ampla distribuição geográfica (RouBIK 2000) e foi amostrada na maioria dos estudos realizados no Brasil (WILMs et al. 1996; RAMALHO 1995). Quando comparamos a abundância obtida para esta espécie em alguns desses estudos, percebemos que aquela aumenta da Mata Atlântica em direção à área central da caatinga (Figura 3). Esses resultados corroboram a teoria proposta por PRICE (1997) de que ambientes com baixa variedade de recursos alimentares tendem a favorecer o estabelecimento de poucas espécies de insetos com populações numerosas; enquanto ambientes com grande variedade de recursos - p. ex., Mata Atlântica (Ramalho 1995) - tendem a ter maior riqueza de espécies de populações pequenas.

A variação observada na abundância mensal das abelhas eussociais durante o período amostral pode ser o resultado da preferência das mesmas por plantas muito produtivas. Sendo assim, pode-se inferir que, durante o período de escassez de recursos altamente produtivos na área, essas abelhas se deslocariam para áreas adjacentes onde esses recursos estariam sendo oferecidos; do mesmo modo, populações de outras localidades seriam atraídas às flores da área amostrada nos períodos de maior disponibilidade desses recursos na mesma. Essa mobilidade das populações, aliada ao fato de ter chovido no mês de setembro, o que estimulou a floração de algumas espécies vegetais chaves (p. ex., Byrsonima correifolia Adr. Juss., Malpighiaceae), pode explicar, por exemplo, o aumento da abundância de abelhas no mês de outubro.

Experimentos de laboratório realizados por SEeley (1985) revelaram que nas regiões temperadas, A. mellifera inicia a produção de crias no meio do inverno para que a colônia esteja suficientemente populosa para enxamear nos primeiros períodos de dias longos, coincidindo com o aparecimento das primeiras floradas. Na caatinga, comportamento semelhante parece ocorrer no final do período seco. Em Milagres, CASTRO (2001) analisou o fluxo quantitativo de pólen coletado por $A$. mellifera e registrou um aumento da quantidade de pólen coletado nos meses que antecedem o início das chuvas, com o pico de coleta coincidindo com o início das precipitações.

Nas DMRSF, os dados de coletas nas flores e as

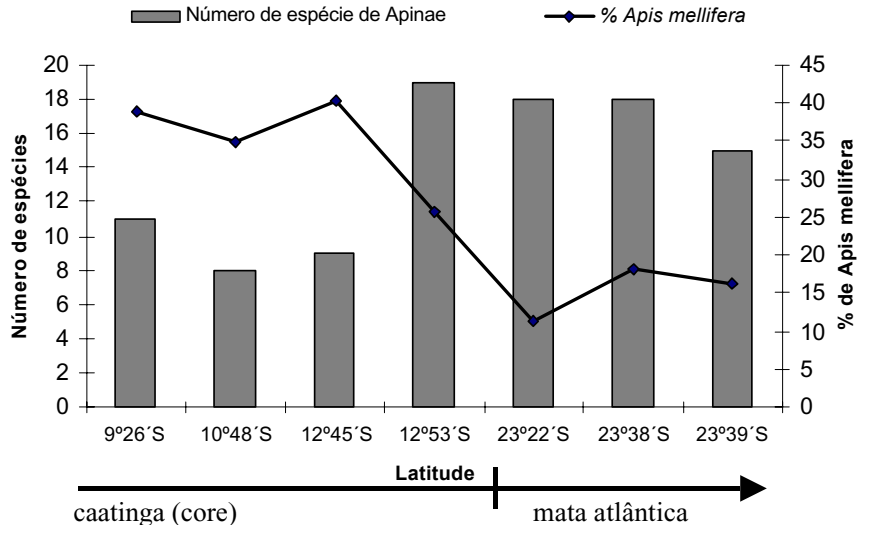

Fig. 3. Riqueza de espécies de Apinae e percentagem de A. mellifera registrados em alguns trabalhos melissocenóticos realizados no Brasil. Dados extraídos, de Martins (1994) em Casa Nova-BA, Viana (1999) em Ibiraba-BA, CARvalho (1999) em Castro Alves-BA, Castro (2001) em Milagres-BA, Ramalho (1995) na Cantareira-SP, Wilms et al. (1996) na Boracéia-SP, Aguilar (1998) no Morro Grande-SP, respectivamente.

observações do ninho nos levam a inferir que a alta abundância de $A$. mellifera no final do período seco foi devido a uma intensificação na produção de crias para (1) garantir a formação de uma população numerosa capaz de enxamear no início da estação de chuvas; (2) atingir grandes populações no início do período de chuvas para forragear uma maior quantidade de recursos e armazenar esses recursos para os períodos de escassez de alimento e/ou (3) porque as primeiras chuvas - que ocorrem ainda no período seco - são um estímulo natural para que as colônias intensifiquem a produção de crias.

Muitas plantas tropicais secretam néctar durante as horas frias da manhã, ainda escuro, e A. mellifera pode explorar esses recursos porque voa a baixas temperaturas e a baixos níveis de luminosidade (SEELEY 1985). Na caatinga, a combinação de disponibilidade de recursos e condições meteorológicas adversas (alta temperatura e baixa umidade relativa) parece favorecer a concentração da atividade de visitação floral por essas abelhas durante a manhã (AguiAr \& MARTins 1997; VIANA 1999). A. mellifera e T. spinipes iniciaram suas atividades de coleta diária às $4: 20 \mathrm{~h}$ e $5: 20 \mathrm{~h}$, respectivamente e permanecem mais ativas até a 10:00h; essa estratégia de forrageamento possui três possíveis vantagens: (1) evita que as abelhas sofram com a alta temperatura e a baixa umidade do ar; (2) possibilita a coleta de recursos tróficos não alterados. O néctar, por exemplo, pode aumentar sua concentração devido à evaporação ao longo do dia (PRoctor et al. 1996); (3) encontram maior quantidade de alimento, fator limitante para essas abelhas que possuem colônias muito populosas e ativas durante todo o ano. Melipona asilvai e M. mandacaia também foram abundantes durante o período da manhã, provavelmente devido aos dois primeiros fatores.

A aclimatação para altas temperaturas parece ser imprescindível à sobrevivência dos insetos em condições naturais, pois as altas temperaturas sazonais e diárias são 
Dunas de Ibiraba-BA (Trabalho atual)

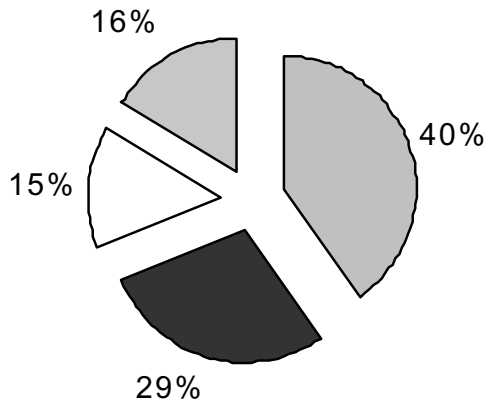

Castro Alves-BA (CARVALHO 1999) $8 \%$

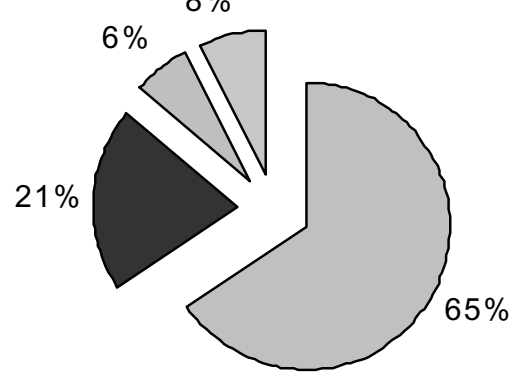

S. J. Cariri-PB (AGUIAR \& MARTINS 1997)

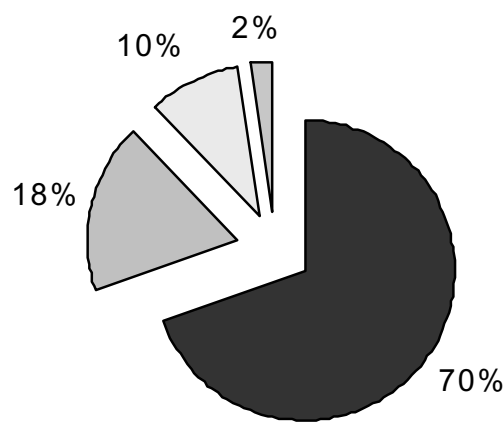

Milagres-BA (CASTRO 2001)

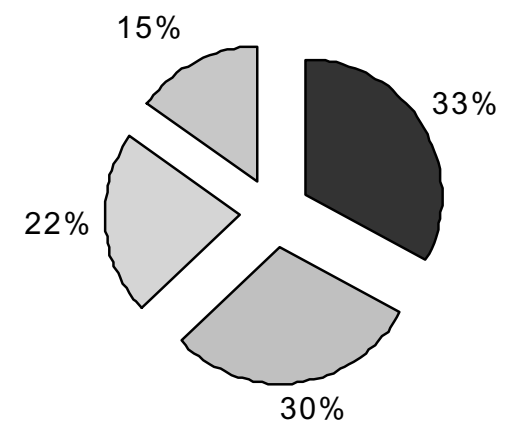

Dunas de Ibiraba-BA (VIANA 1999)

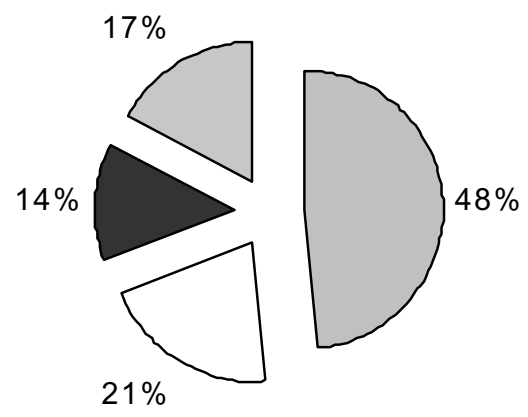

Casa Nova-BA (CASTRO 1994)

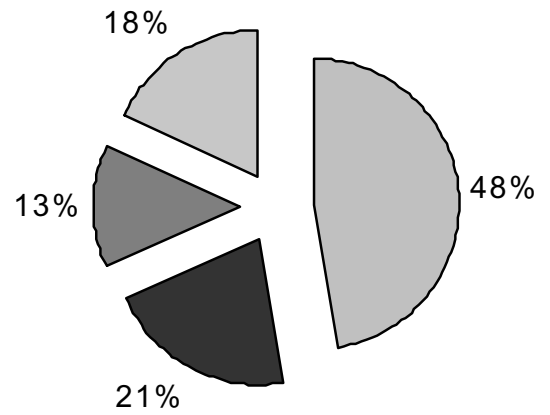

Fig. 4. Espécies de abelhas eussociais predominantes nas cinco localidades estudadas na caatinga.

T. spinipes;
A. mellifera; F. s. languida; Trigonisca sp.;
F. doederleini;
Scaptotrigona tubiba;
T. fuscipennis;

Outros.

precedidas por um gradiente de temperaturas mais baixas, e vice-versa (Romoser \& Stoffolano JR. 1994). SAKagami \& ZuCCHI (1974), estudando ninhos de Trigonisca duckei Friese (1900), observaram que essa espécie tem pouca capacidade de termorregulação, paralisando completamente suas atividades em dias frios e à noite. Em Ibiraba, Trigonisca sp. iniciou suas atividades a partir das 8:20h, o que nos leva a inferir que essa espécie precisa de um período de aclimatação de cerca de 3 a $4 \mathrm{~h}$, a partir do alvorecer para iniciar as atividades de forrageio, alcançando seu pico de atividade no intervalo de 10:00 às 12:00h, quando são registradas altas temperaturas. Nesse caso, a diminuição do tempo útil de coleta é compensada pela capacidade de transportar maior quantidade de pólen (RAMALHO et al. 1994), possivelmente devido à maior área relativa da corbícula em relação à área e à massa corporal (FRANÇOSO JR. \& BICUDo 2000). Essas abelhas parecem não sofrer o impacto da depleção dos recursos tróficos ao longo do dia devido ao pequeno tamanho de suas populações, que necessitam de pequena quantidade de alimento.

Comportamento semelhante foi observado para $F$. $s$. languida, mas com intervalos menores. RIBEIRO \& BEGO (1994) observaram em laboratório que operárias de $F$. s. languida moviam-se lentamente a temperaturas entre 26 e $28^{\circ} \mathrm{C}$ e nos dias mais frios cessavam totalmente suas atividades, inclusive 


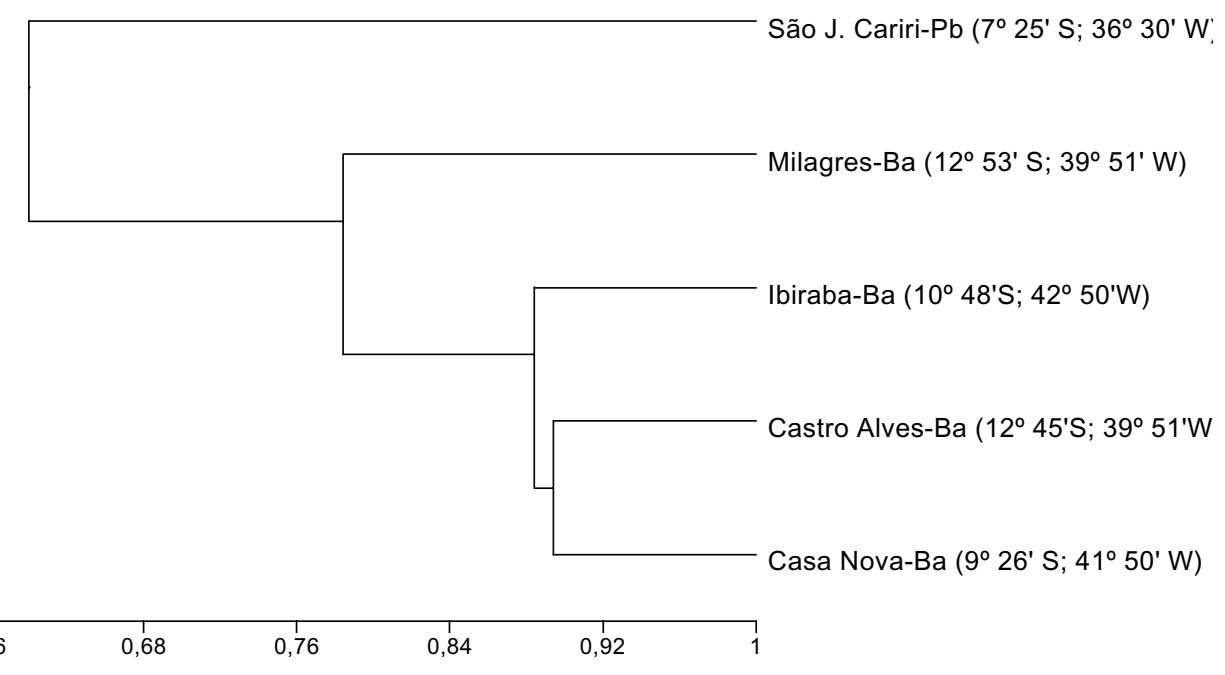

Fig. 5. Similaridade entre as faunas de abelhas eussociais de cinco localidades estudadas na caatinga. Dados extraídos de AguiAR \& MARTins (1997) em S. J. Cariri-PB, CAstro (2001) em Milagres-BA, trabalho atual em Ibiraba-BA, CARvalho (1999) em Castro Alves-BA e Martins (1994) em Casa NovaBA.

a construção de células e postura. As abelhas só voltavam às suas atividades normais a temperaturas superiores a $31^{\circ} \mathrm{C}$.

Na região tropical, em períodos de clima desfavorável, os insetos tendem a reduzir seu metabolismo (entrar em dormência) ou migrar para áreas de clima mais favorável para resistir às adversidades ambientais (SAUNDERs 1982). Ao que parece, as abelhas podem utilizar duas estratégias diferentes para resistir às irregularidades bióticas e abióticas da caatinga: diapausa pré-pupal e/ou migração do ninho.

As abelhas eussociais nas DMRSF não entraram em diapausa durante seu ciclo vital, já que os ninhos permaneceram ativos durante todo o período amostral. Abandono de ninho em direção às margens dos rios permanentes parecem ser os mecanismos utilizados pela $A$. mellifera para sobreviver aos períodos de estresse da caatinga (Aguiar \& Martins 1997; CAStro 2001), que pode estar mais relacionado com a disponibilidade de água para a termoregulação da colônia do que à disponibilidade de alimento, já que essas abelhas armazenam alimento para os períodos de escassez.

$\mathrm{Na}$ literatura existem poucos registros sobre o abandono de ninhos por Meliponini (RoubiK 1979; Ribeiro \& BeGo 1994). CAStro (2001) registrou a morte de toda uma população de $M$. asilvai e Melipona subnitida Ducke, 1910 (32 colônias) na estação seca em Milagres, sugerindo que essas espécies não abandonam seus ninhos nem mesmo nos períodos de maior escassez de recursos.

De modo geral, a comunidade de abelhas eussociais das DMRSF apresenta um padrão de organização semelhante aos observados nas outras localidades estudadas na caatinga, mas com algumas particularidades resultantes da organização estrutural diferenciada das dunas, conforme discutido no presente trabalho.
Agradecimentos. À CAPES pela concessão da bolsa de mestrado ao primeiro autor; ao $\mathrm{CNPq}$ pela concessão da bolsa de produtividade à pesquisa ao segundo autor; ao CNPq e PRODOC/UFBA/CADCT pelo auxílio financeiro dado ao projeto; a Favízia F. de Oliveira (UFPR) pela identificação de F. doederleini; a Ana T. A. Rodarte (IB-UFBA) pela coleta de dados das espécies vegetais; à equipe do LABEA pelo auxílio nas atividades de campo; ao INMET- IV Distrito pelo fornecimento dos dados meteorológicos.

\section{REFERÊNCIAS}

Aguiar, C. M. L.; C. F. Martins \& A. C. A Moura. 1995. Recursos florais utilizados por abelhas (Hymenoptera, Apoidea) em área de caatinga (São João do Cariri, Paraíba). Revista Nordestina de Biologia 10(2): 101-117.

Aguiar, C. M. L. \& C. F. Martins. 1997. Abundância relativa, diversidade e fenologia de abelhas (Hymenoptera, Apoidea) na caatinga, São João do Cariri, Paraíba, Brasil. Iheringia, Série Zoologia 83: 151-163.

Agullar, J. B. V. 1998. A Comunidade de Abelhas (Hymenoptera, Apoidea) da Reserva Florestal Morro Grande, Cotia, São Paulo. Tese de Doutorado, Instituto de Biociências, Universidade de São Paulo, 80 p.

Carvalho, C. A. L. 1999. Diversidade de abelhas (Hymenopetra, Apoidea) e plantas visitadas no município de Castro Alves, BA. Tese de Doutorado, Escola Superior de Agricultura Luiz de Queiroz, Universidade de São Paulo, 104p.

Castro, M. S. 1994. Composição, fenologia e visita às flores pelos Apidae em Casa Nova, Bahia. Dissertação de Mestrado, Instituto de Biociências, Universidade de São Paulo, 103p.

Castro, M. S. 2001. A Comunidade de abelhas (Hymenopetra, Apoidea) de uma área de caatinga arbórea entre os inselbergs de Milagres $\left(1^{\circ} 53^{\prime} \mathrm{S} ; 3^{\circ} 51^{\prime} \mathrm{W}\right)$, Bahia. Tese de Doutorado, Instituto de Biociências, Universidade de São Paulo, 191p.

De Oliveira, P. E.; A. M. F. Barreto \& K. Suguio. 1999. Late Pleistocene/ Holocene climatic and vegetational history of the Brazilian caatinga: 
the fossil dunes of the middle São Francisco River. Paleogeography, Paleoclimatology, Paleocology 152: 319-337.

Françoso Jr., O. A. \& J. E. P. W. Bicudo. 2000. Estudo comparativo do metabolismo aeróbio de Melipona bicolor Lepeletier (Hymenoptera: Apidae: Meliponinae). In: Anais do IV Encontro sobre Abelhas de Ribeirão Preto, p. 86-93.

Laroca, S. 1995. Ecologia: Princípios e Métodos. Petrópolis, Ed. Vozes, 197p.

Ludwig, J. A. \& J. F. Reynolds. 1988. Statistical Ecology: a Primer on Methods and Computing. New york, John Wiley \& Sons, 337p.

Magurran, A. E. 1988. Ecological Diversity and its Measurement. Princeton, Princeton University Press, 179p.

Martins, C. F. 1994. Comunidade de abelhas (Hymenoptera: Apoidea) da caatinga e do cerrado com elementos de campos rupestres do estado da Bahia, Brasil. Revista Nordestina de Biologia 9: 225-257.

Michener, C. D. 1979. The Social Behavior of the Bees - A Comparative Study. Cambridge, Harvard University, 404p.

Neves, E. L. \& B. F. Viana. 1999. Comunidade de Euglossinae (Hymenoptera, Apidae) das matas ciliares da margem esquerda do médio Rio São Francisco, Bahia. Anais da Sociedade Entomológica do Brasil 28(2): 201-210.

Pielou, E. C. 1975. Ecological Diversity. New York, John Wiley \& Sons, $165 \mathrm{p}$.

Pinheiro-Machado, C.; I. Alves dos Santos; F. A. Silveira; A. M. P. Kleinert \& V. L. Imperatriz-Fonseca. 2002. Brazilian bee surveys: state of knowledge, conservation and sustainable use. In: P. G. KeVAN; V. L. Impreatriz-Fonseca; G. W. Frankie; C. O'Toole; C. H. Vergana \& T. Feltz (eds.). Pollinating Bees: a link between agriculture and conservation. Brasília, Barbara Bela Editora.

Price, P. W. 1997. Insect Ecology. 3rd ed. New York, John Wiley \& Sons, $874 \mathrm{p}$.

Proctor, M.; P. Yeo \& A. Lack. 1996. The Natural History of Pollination. London, Haper Collins Publishers, 479p.

Ramalho, M; T. C. Giannini \& K. S. Malagodi-Braga. 1994. Pollen harvest by stingless bee foragers (Hymenoptera, Apidae, Meliponinae). Grana 33: 239-244.

Ramalho, M. 1995. Diversidade de Abelhas (Apoidea, Hymenoptera) em um remanescente de floresta atlântica, em São Paulo. Tese de Doutorado, Instituto de Biociências, Universidade de São Paulo, 144p.
Ribeiro, M. \& L. R. Bego. 1994. Absconding in the Brasilien Stingless Bee Frieseomelitta silvestri languida Moure (Hymenoptera: Apidae: Meliponinae). Anais da Sociedade Entomológica do Brasil 23(2): 3550-3558.

Romoser, W. S. \& J. G. Stoffolano Jr. 1994. The Science of Entomology. 3th ed. Dubuque, Brown Communications, 532p.

Roubik, D. W. 1979. Nest and colony characteristics of stingless bees from French Guiana (Hymenoptera: Apidae). Journal of the Kansas Entomological Society 52(3): 443-470.

RoubiK, D.W. 1989. Ecology and natural history of tropical bees. New York, Cambridge University Press, 314p.

RouBiK, D. W. 2000. The foraging and potential outcrossing pollination ranges of african honey bees (Apiformes: Apidae; Apini) in Congo forest. Journal of the Kansas Entomological Society 72(4): 394-401.

Sakagami, S. F. \& R. Zucchi. 1974. Oviposition of two dwarf stingless bees, Hypotrigona (Leurotrigona) muelleri and H. (Trigonisca) $d u c k e i$, with notes of oviposition process in stingless bees. Journal of the Faculty of Hokkaido University VI, Zoology 19(2): 361421.

Sakagami, S. F.; S. Laroca \& J. S. Moure. 1967. Wild bee biocenotics in São José dos Pinhais (PR), South Brazil. Preliminary Report. Journal of the Faculty of Hokkaido University VI, Zoology 19: 190250 .

Saunders, D. S.1982. Insect Clock. 2nd ed. Oxford, Pergamon Press, $409 \mathrm{p}$.

Seeley, T. D. 1985. Honeybee Ecology. Princenton, Princeton University Press, $201 \mathrm{p}$.

Teixeira, A. F. R. \& B. F. Viana. 2002. Distribuição e densidade dos sítios nidificados pelos meliponíneos (Hymenoptera:Apidae) das Dunas do Médio São Francisco, Ibiraba, Barra-Bahia. Revista Nordestina de Zoologia 2 (1):

Viana, B. F. 1999. A Comunidade de abelhas (Hymenoptera, Apoidea) das Dunas Interiores do Rio São Francisco, Bahia, Brasil. Anais da Sociedade Entomológica do Brasil 28(4): 635-645.

Wilms, W.; V. L. IMPERATRIZ-FonsECA \& W. Engels. 1996. Resource partioning between highly eusocial bees and possible impact of the introduced Africanized honey bee on native stingless bees in the brasilian atlantic rainforest. Studies on Neotropical Fauna and Environment 31: $137-151$. 